\title{
Long Term Variations in Magnetotelluric Apparent Resistivities Observed near the San Andreas Fault in Southern California
}

\author{
Barry R. Lienert, ${ }^{*}$ James H. Whitcomb, ${ }^{*,+}$ R. J. Phillips, ${ }^{* *}$ \\ I. K. REDDY, ${ }^{\dagger \dagger}$ and R. A. TAYLOR* \\ *Seismological Laboratory, California Institute of Technology, \\ Pasadena, California, U.S.A. \\ **Jet Propulsion Laboratory, 4800 Oak Grove Drive, Pasadena, \\ California, U.S.A.
}

(Received September 10, 1980)

Since early 1975, we have monitored magnetotelluric apparent resistivities at three sites close to the Los Angeles metropolitan area. We estimate the repeatability of our calculated apparent resistivities to be $10-20 \%$. A major problem has been the removal of bias in the resistivity estimates caused by cultural noise, mainly on the magnetic field components. We have attempted to remove this bias using a variety of techniques including singular value decomposition of the signal matrix. None of these procedures was entirely successful when the signal predictabilities fell below $80 \%$.

The only major earthquake to occur in our study area was the Santa Barbara shock on August 13, $1978\left(M_{L}=5.2\right)$. No significant changes were observed in apparent resistivities measured at a site situated $80 \mathrm{~km}$ to the east of the epicenter both 25 days before and 7 days after this shock. Significant changes in low frequency apparent resistivities were observed at a site in West Antelope Valley, $50 \mathrm{~km}$ northwest of Palmdale. Between April 1976 and July 1978 the rotated minimum apparent resistivities in the frequency range $0.001-0.1 \mathrm{~Hz}$ increases $10-15 \%$, while the maximum resistivities remained almost constant. The direction of the maximum rotated apparent resistivity also changed by as much as $20^{\circ}$. These changes cannot be correlated with any obvious change in seismicity. However, they may be related to the uplift and subsidence which is known to have occurred in this area.

We have also observed changes in the high frequency $(>1 \mathrm{~Hz})$ apparent resistivities following a heavy rainfall which occurred in the spring of 1978 . At one site, the apparent resistivities at $10 \mathrm{~Hz}$ dropped two orders of magnitude. This effect was modelled by adding a surface layer with a thickness of $10 \mathrm{~m}$ and a resistivity of $10 \Omega \mathrm{m}$.

$\dagger$ Present address: CIRES, University of Colorado, Boulder, Colorado, U.S.A.

tt Present address: Shell Oil Co., Houston, Texas, U.S.A. 


\section{Introduction}

Laboratory studies of the electrical resistivities of water saturated crystalline rocks as a function of applied stress have indicated that their resistivities may drop by as much as an order of magnitude before fracture occurs (BRACE and ORANGE, 1968). Electrical resistivity sounding techniques such as the magnetotelluric method should therefore be sensitive to the large scale changes in tectonic stress patterns which precede large earthquakes. Actual drops in resistivity preceding earthquakes have been observed by BARSUKOV (1972) in the U.S.S.R. and MAZZELA and MoRrISON (1974) in central California.

The work described in this paper is an extension of a study of long-term variations in magnetotelluric apparent resistivities at three sites close to the Los Angeles metropolitan area in Southern California, U.S.A. (REDDY et al., 1976). The results from the present study extend the period of coverage from the beginning of 1975 up to the end of 1979 .

The locations of the three sites which we have been monitoring appear in Fig. 1, taken from REDDY et al. (1976). The solid dot on the map represents the epicenter of the largest earthquake which occurred during the study, the Santa Barbara Channel event of August 13, $1978\left(M_{L}=5.2\right)$.

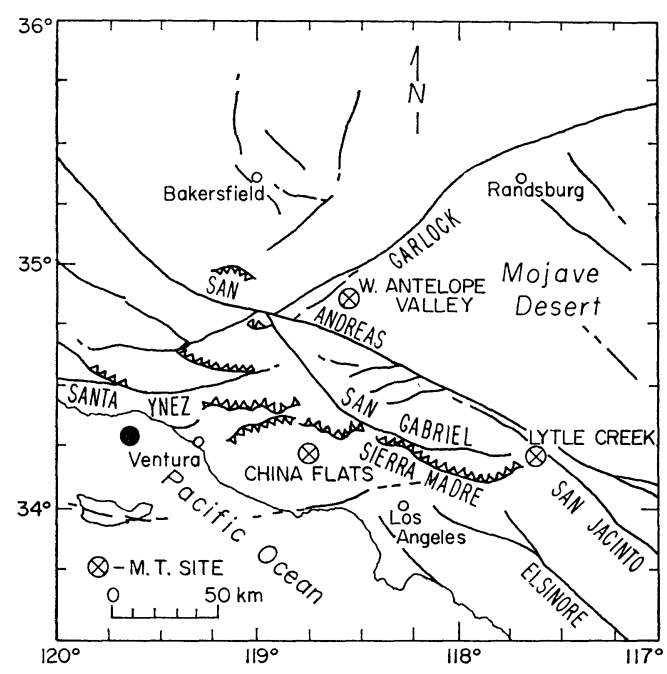

Fig. 1. Map of the Southern California region showing the locations of the three magnetotelluric sites relative to the major faults. The solid dot represents the epicenter of the magnitude 5.2 earthquake which occurred August 13, 1978. 


\section{Instrumentation}

Prior to 1977, the instrumentation incorporated induction-type magnetic field sensors similar to those described by Allsopp et al. (1974). This system is described in detail by REDDY et al. (1976). Commencing in 1977, a new data acquisition system was used which incorporated a three-component S.H.E. cryogenic magnetometer. Unfortunately, a variety of problems with this new system prevented usable data from being obtained until July of 1978, after major modification had been made to both the analog and digital sections of the electronics. A block diagram of the present system appears in Fig. 2.

Electric fields are monitored as voltages between three copper electrodes arranged in a single-ended " $L$ " configuration with the corner electrode as common ground. These two voltages, along with the output voltages of the magnetometer electronics are suitably amplified by variable band-width preamplifiers having maximum gains of 80 $\mathrm{dB}$ for the electric fields and $40 \mathrm{~dB}$ for the magnetic fields. Further amplification of up to $18 \mathrm{~dB}$ is accomplished in post-amplifiers which incorporate variable frequency low-pass alias filters.

Each site was normally occupied for at least one complete evening. Data was recorded in three overlapping frequency bands, $0.001-0.2 \mathrm{~Hz}, 0.01-1 \mathrm{~Hz}$, and $0.5-$ $30 \mathrm{~Hz}$ using digital sampling rates of $0.5,5$, and 100 samples/sec, respectively.

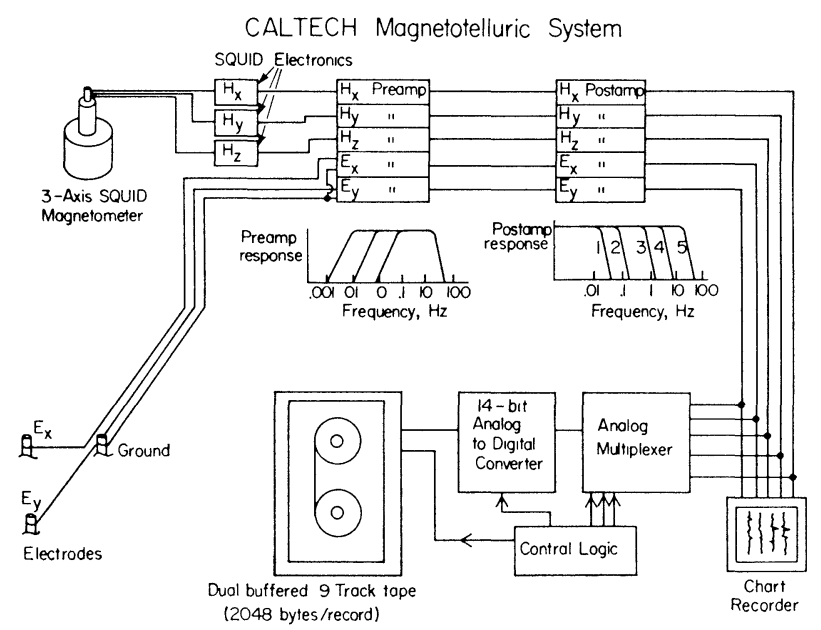

Fig. 2. Block diagram of the magnetotelluric system used in collecting the 1978-1979 data.

\section{Data Analysis}

Segments of the recorded time series from each frequency band 2048 points long were first of all selected by visually inspecting plots of the digitally recorded data. 
To remove undesirable peaks in the low frequency power spectra, a high pass digital recursive filter was applied to each time series. The data were then transformed into the frequency domain using the Fast Fourier Transform algorithm. Auto and crosspower spectral estimates were obtained by multiplication of the complex Fourier coefficients at each frequency after smoothing with a Goodsman-Enochson window (OTNES and ENOCHSON, 1972). The resulting spectral estimates were then averaged over frequency bands having a $Q$ of 10 . In the lowermost decade of frequency, the $Q$ was reduced to improve the statistical stability of the estimates. Smoothed spectral estimates were normally obtained for at least three separate segments of data with the results being averaged for each frequency band.

The next stage in the analysis involves estimating the complex tensor elements which relate electric to magnetic fields at each frequency, which are defined by the linear relations:

$$
\begin{aligned}
& E_{x}=Z_{x x} H_{x}+Z_{x y} H_{y} \\
& E_{y}=Z_{y x} H_{x}+Z_{y y} H_{y} .
\end{aligned}
$$

$E_{x}$ and $E_{y}$ are the complex spectral amplitudes of the horizontal electric fields in the $X$ and $Y$ directions, $H_{x}$ and $H_{y}$ are the magnetic field amplitudes and $Z_{x x}, Z_{x y}$, $Z_{y x}, Z_{y y}$ are the elements of the impedance tensor at the given frequency. Solutions for the elements of the impedance tensor can then be obtained in terms of auto and cross-spectral estimates in a given frequency band (SwIFT, 1967; VozofF, 1972), namely,

$$
Z_{x x}=\frac{\left\langle E_{x} A^{*}\right\rangle\left\langle H_{y} B^{*}\right\rangle-\left\langle E_{x} B^{*}\right\rangle\left\langle H_{y} A^{*}\right\rangle}{\left\langle H_{x} A^{*}\right\rangle\left\langle H_{y} B^{*}\right\rangle-\left\langle H_{x} B\right\rangle\left\langle H_{y} A^{*}\right\rangle}
$$

$\mathrm{A}$ and $\mathrm{B}$ are any two of the components $E_{x}, E_{y}, H_{x}, H_{y}$, the brackets represent spectral averages over a given frequency band, while the asterisks represent complex conjugates. As Sims et al. (1971) have observed, this solution is biased by the presence of noise on the components chosen as $\mathrm{A}$ and $\mathrm{B}$ (usually $H_{x}$ and $H_{y}$ ) as it only minimizes noise on the other two components. Two important quantities used in checking for the presence of such noise are the predictabilities of the $E_{x}$ and $E_{y}$ components. These are defined as the coherence between the predicted values of $E_{x}$ and $E_{y}$, obtained by substituting the measured values of $H_{x}$ and $H_{y}$ into Eq. (1), and the measured values of $E_{x}$ and $E_{y}$. They will have a maximum value of one when all components are noise-free.

The resulting estimates of the tensor impedances are then rotated into the direction which minimizes the sum $\left|Z_{x x}\right|^{2}+\left|Z_{y y}\right|^{2}$ (VozofF, 1972). This direction was determined by rotating the impedances through $5^{\circ}$ intervals and calculating the above sum at each step. The rotated values of the tensor elements $Z_{x y}$ and $Z_{y x}$ can then be converted to minimum and maximum apparent resistivities, $\rho_{\max }$ and $\rho_{\min }$, by the use 
of the formulae

$$
\rho_{\max }=0.2 T\left|Z_{x y}^{\prime}\right|^{2}
$$

and

$$
\rho_{\min }=0.2 T\left|Z_{x y}^{\prime}\right|^{2} .
$$

$Z_{x y}^{\prime}$ and $Z_{y x}^{\prime}$ are the rotated values of the tensor impedances in $\mathrm{mVnT}^{-1} \mathrm{~km}^{-1}$ and $T$ is the center period of the frequency band in sec.

To calculate errors in the impedances, we have used a method described earlier (REDDY et al., 1976). This method assumes that errors in the individual components are random and that the resulting tensor impedances follow an $F$-distribution (BENDAT and PIERSOL, 1971). The spread of this $F$-distribution depends on the relative magni , tudes of the auto-powers, the number of degrees of freedom in the estimate, the predictabilities of $E_{x}$ and $E_{y}$ and the coherency between $H_{x}$ and $H_{y}$.

\section{Bias Removal}

Inevitably, much of the data we have collected in this study is biased by the effect of noise, mainly of cultural origin, on both electric and magnetic fields. It is therefore essential to incorporate some type of bias removal into the analysis. The technique we have adopted is to estimate the signal to noise ratios on each of the four components using relationships between signal to noise and multiple coherence (predictability) (WhITE, 1973), i.e.,

$$
\begin{aligned}
& \gamma_{312}^{2}=\left(\frac{\rho_{3}}{1+\rho_{3}}\right)\left(\frac{\rho_{2}+\rho_{1}}{1+\rho_{2}+\rho_{1}}\right) \\
& \gamma_{412}^{2}=\left(\frac{\rho_{4}}{1+\rho_{4}}\right)\left(\frac{\rho_{1}+\rho_{2}}{1+\rho_{1}+\rho_{2}}\right) \\
& \gamma_{134}^{2}=\left(\frac{\rho_{1}}{1+\rho_{1}}\right)\left(\frac{\rho_{4}+\rho_{3}}{1+\rho_{4}+\rho_{3}}\right) \\
& \gamma_{234}^{2}=\left(\frac{\rho_{2}}{1+\rho_{1}}\right)\left(\frac{\rho_{4}+\rho_{3}}{1+\rho_{4}+\rho_{3}}\right) .
\end{aligned}
$$

$\rho_{i}$ is the signal to noise ratio on the $i^{\text {th }}$ component where $H_{x}=1, H_{y}=2, E_{x}=3, E_{y}=4$, while $\gamma_{i j k}$ is the multiple coherence (predictability) of the $i^{\text {th }}$ component in terms of the $j^{\text {th }}$ and $k^{\text {th }}$ components. The four multiple coherencies on the left hand sides of Eqs. (3)-(6) can readily be calculated from the auto and cross power spectral averages as described by REDDY and RANKIN (1974). We have used the following procedure to solve Eqs. (3)-(6).

(i) Assume $\rho_{1}, \rho_{2} \rightarrow \infty$ (noise free).

(ii) Solve (3) and (4) for $\rho_{3}$ and $\rho_{4}$. 

(ii).

(iii) Solve (5) and (6) for $\rho_{1}$ and $\rho_{2}$ using the values of $\rho_{3}$ and $\rho_{4}$ obtained in step

(iv) Iterate steps (ii) and (iii). If the first iteration does not give a solution for $\rho_{1}$ and $\rho_{2}$, these steps are repeated commencing with successively smaller finite values of $\rho_{1}$ and $\rho_{2}$.

(v) Calculate corrected auto-powers using the relations

$$
\begin{gathered}
P_{i, \text { corrected }}=P_{i, \text { measured }} \frac{\rho_{i}}{1+\rho_{i}} \\
i=1 \ldots 4
\end{gathered}
$$

where $P_{i}$ is the auto-power of the $i^{\text {th }}$ component.

(vi) Recalculate the multiple coherencies using the corrected values of the autopowers.

(vii) Iterate steps (i)-(vi) until any of the multiple coherencies become equal to or exceed 1.

(viii) Use the corrected values of the magnetic field auto-powers, $P_{1}$ and $P_{2}$ in Eq. (2) with $A=H_{x}, B=H_{y}$ to obtain corrected values of the tensor impedances in the given frequency band. In this manner, noise on the $E$ fields is removed by the least squares minimization while $H$ noise is removed by the above procedure.

A more elegant method of removing bias in impedance tensor estimates has been described by JupP (1978). In his method, all the individual estimates of the 5 components in a given frequency band are represented by an $M \times 5$ complex matrix, where $M$ is the total number of estimates. This complex matrix is then decomposed into signal and noise matrices by singular value decomposition. The result is a set of $M$ eigenvalues and their corresponding eigenvectors, the largest two of these being used to calculate the signal matrix. Effectively, this simultaneously minimizes the noise on all 5 field components where the noise is by definition any part of each component which is not linearly related through the tensor Eq. (1).

We applied this technique to a large quantity of the data collected during 1978 and 1979. The general conclusions that we have made regarding its usefulness are:

(i) Weighting the data in each frequency band with a triangular (Parzen) window, as suggested by JUPP (1978) results in such a severe loss in the number of degrees of freedom that the technique becomes ineffective.

(ii) Including the vertical magnetic field component in the analysis did not significantly improve the results. This is probably due to the rather poor correlation of vertical to horizontal magnetic fields at all three of our sites.

(iii) The technique was most successful when the signal to noise ratios on the components were all less than about $15 \%$. A large amount of noise on any one component severely biased the resulting estimates. We attempted to remedy this by weighting the noisy components as suggested by JuPP (1978), but this met with little success. 
(iv) The major value of the method is that it enables noise on a single component to be readily identified. It also can give some insight into the presence of effects such as non-uniform source fields, although this requires high coherencies between the vertical and horizontal magnetic field components.

(v) The method involves a considerable amount of computation. In the higher frequency bands it more than doubled the total computer time required to obtain impedance tensor estimates.

Neither of the bias removal techniques described above was completely successful when applied to large quantities of data. This was undoubtedly due in many cases to noise which was correlated between the electric and magnetic fields. As PRICE (1962) has observed, the magnetotelluric method assumes laterally uniform source fields and this assumption may not always be valid, especially at very low frequencies. It is also probable that some sources of noise on the magnetic field which are of cultural origin (transmission lines, etc.) may induce significant currents to flow in the ground close to a measurement site. The result would be correlated noise on the electric field which would not be completely removed by either of the above techniques.

In this study we have not attempted to use data for which the predictabilities were below 0.85 . The amount of bias removed was then fairly small and did not differ significantly for the two techniques, although JUPP's (1978) technique did appear to be slightly superior in some instances.

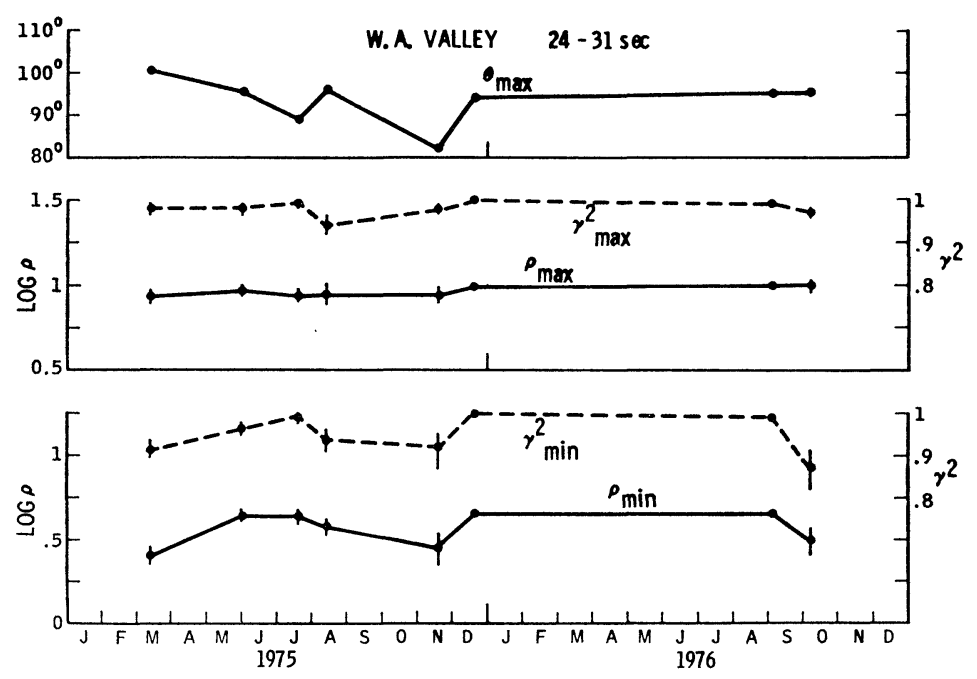

Fig. 3. Plot of the apparent resistivities (ohm-m) $\rho_{\min }$ and $\rho_{\max }$, the squares of their predictabilities, $\gamma$, and the strike of the direction of apparent resistivity, $\theta_{\max }$ for the West Antelope Valley site during 1975-1976. Results were calculated using $A=H_{x}$ and $B=$ $H_{y}$ in Eq. (2). 

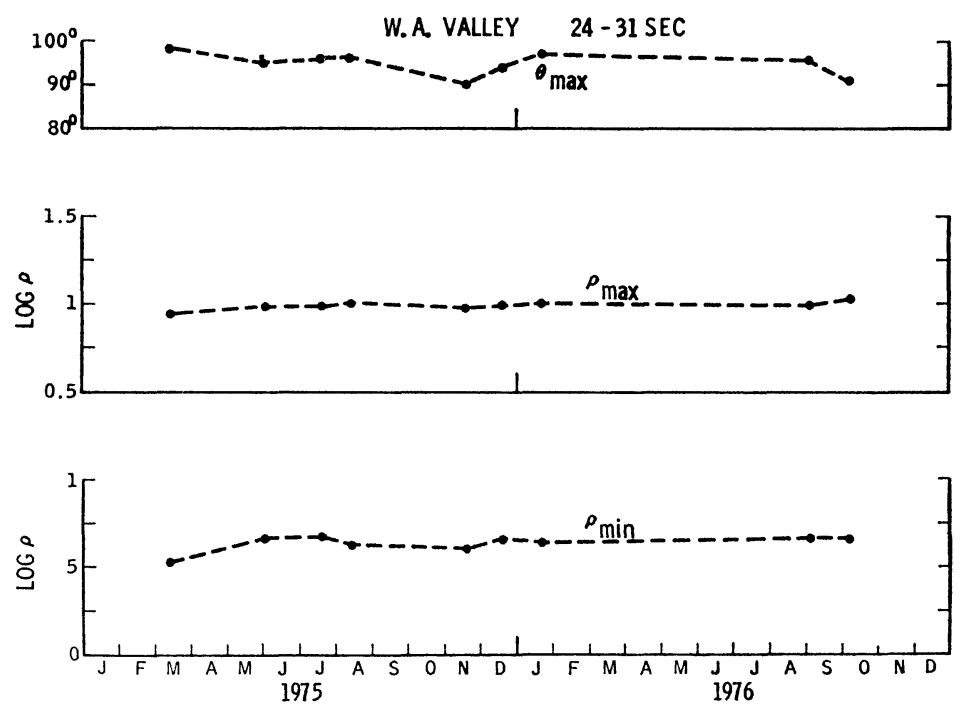

Fig. 4. Plot of results for the same data used in Fig. 3 after application of the bias removal technique described in the text.

Shown in Fig. 3 are the rotated maximum, minimum apparent resistivities and maximum resistivity direction for the 1975-1976 period at the West Antelope Valley. site. These were calculated using conventional $E$-field noise minimization in Eq. (2). Both $\rho_{\max }$ and $\rho_{\min }$ show variations which are strongly correlated with the predictabilities, $\gamma$, reflecting the presence of bias. Figure 4 shows the same data after the application of the multiple coherence bias removal technique. A large amount of the bias has obviously been removed, although a small amount remains on the minimum apparent resistivities. We found that the data could be further improved by leastsquares fitting of fourth order polynomial functions to results from individual frequency bands contained within a one-decade frequency range. Estimates of the apparent resistivities, their phases, direction and upper and lower error limits at the frequency of interest were then obtained from the resulting sets of polynomial functions. This technique proved to be of considerable value, especially when the data from the single frequency band we examined was clearly more biased by noise than the data from adjacent frequency bands.

\section{Results}

\subsection{West Antelope Valley}

This site is located $60 \mathrm{~km}$ northwest of Palmdale, close to the intersection of the San Andreas and Garlock Failts (Fig. 1). Much interest has recently been focused on 
W. A. VALLEY 26 SEC

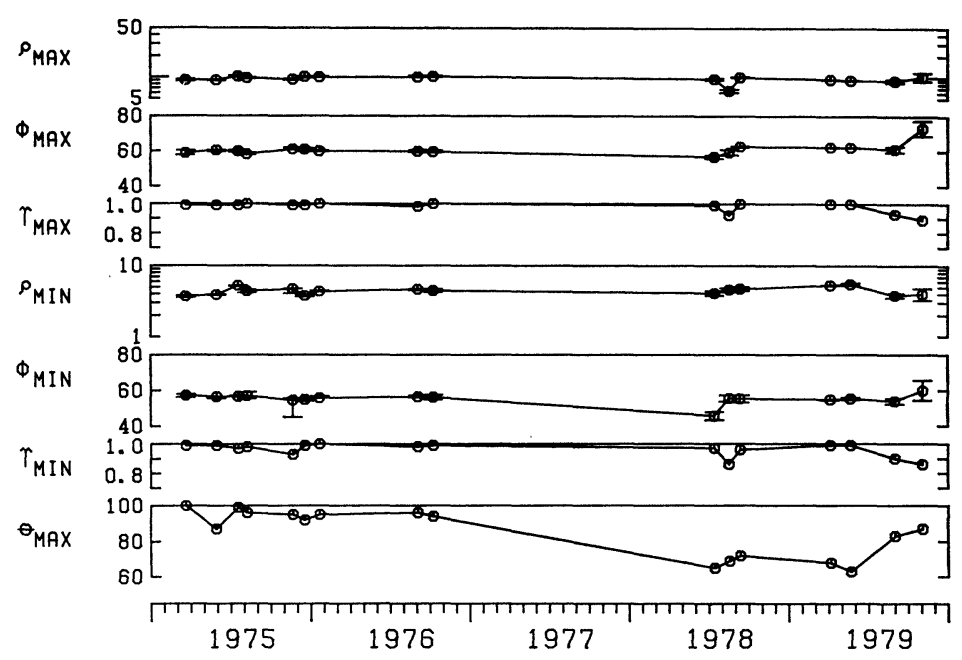

Fig. 5. Variations in apparent resistivities determined at the West Antelope Valley site from March, 1975 to November, 1979. Each of the points was obtained by fitting fourth order polynomials to results obtained within one decade of frequency after application of the multiple coherence bias removal technique to estimates in each of the frequency bands. $\gamma_{\max }$ and $\gamma_{\min }$ are the predictabilities of the electric fields in the directions of maximum and minimum apparent resistivities respectively, while $\phi_{\max }$ and $\phi_{\min }$ are the corresponding phases of apparent resistivities in degrees. $\theta_{\max }$ is the direction (degrees from north) of the maximum apparent resistivity axis.

this area as the initial center of a large scale uplift, popularly termed the "Palmdale Bulge" (CASTLE et al., 1976, 1977). Shown in Fig. 5 are the magnetotelluric data at a period of $26 \mathrm{sec}$ which we have obtained at this site since early 1975 . The most interesting feature in this data is a change in $\theta_{\max }$, the direction of the maximum resistivity axis. This change occurred between the end of 1976 and the middle of 1978 . However, between May and August of 1979, the strike has moved back towards the 1975-1976 direction. At the same time, the minimum apparent resistivity steadily increases from a value of about $3.5 \mathrm{ohm}-\mathrm{m}$ at the beginning of $1975 \mathrm{up}$ to a value of $5 \mathrm{ohm}-\mathrm{m}$ in May of 1979. In August of 1979 the minimum resistivity decreased back to between 3 and 4 ohm-m.

The maximum resistivity remains fairly constant at between 9 and $10 \mathrm{ohm}-\mathrm{m}$ apart from a single value in August, 1978, which is clearly associated with low coherencies and probably due to unremoved bias in the estimate. Variations in the phases of the minimum and maximum resistivities do occur, but appear to be confined to isolated points, and consequently should be viewed with some suspicion.

To show the changes in more detail, we have plotted the complete apparent re- 

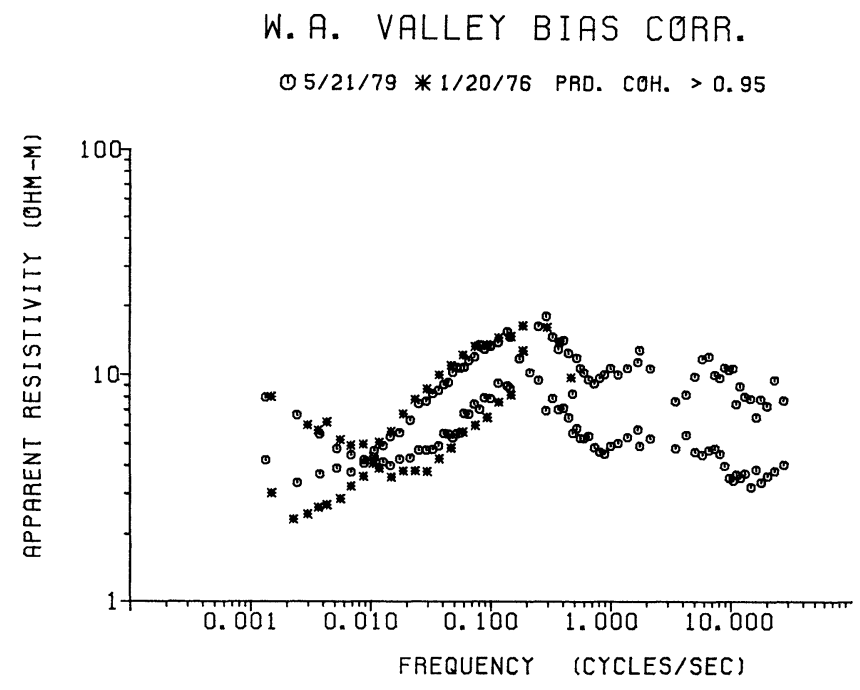

Fig. 6. Bias corrected apparent resistivity data from individual frequency bands obtained in both January, 1976 and May, 1979. The same symbols have been used for both maximum and minimum apparent resistivities. Errors do not appear on this plot but were in almost all cases less than $10 \%$.

sistivity curves obtained both in January, 1976 and in May, 1979 in Fig. 6. The minimum resistivities in the frequency range $0.001-0.01 \mathrm{~Hz}$ measured in 1976 are all lower than those measured in 1979. The effect is most pronounced below the crossover point at $0.01 \mathrm{~Hz}$, where the difference in the two sets of minimum resistivities exceeds $30 \%$. Shown in Fig. 7 are the directions of maximum apparent resistivity, for which the off-diagonal elements of the tensor impedances are minimized. Results were obtained from two sequential months data, both in 1975-1976 and in 1979. Between 0.01 and $0.1 \mathrm{~Hz}$, the directions for the $1975-1976$ data lie about $30^{\circ}$ to the east of those for the 1979 data. Below $0.01 \mathrm{~Hz}$, the situation is reversed with the 1979 directions lying to the east of the 1975-1976 results.

It is rather difficult to draw definitive conclusions from these results due to their obviously three-dimensional nature. The frequency at which the maximum and minimum resistivities cross is about $0.01 \mathrm{~Hz}$, which corresponds to a skin depth of $16 \mathrm{~km}$ in $10 \mathrm{ohm}-\mathrm{m}$ material. The direction of maximum resistivity is not at right angles to either the San Andreas or Garlock Faults as one might expect for two-dimensional structures associated with these faults. However, it may be of significance that the values of $\theta_{\max }$ above $0.1 \mathrm{~Hz}$ moved into a direction more nearly at right angles to the San Andreas fault between 1976 and 1978.

The leveling data of CASTLE et al. (1977) have indicated that the region around Palmdale was uplifted by as much as $20 \mathrm{~cm}$ during 1959 and the early 1960 's. Com- 


\section{W. A. VALLEY BIAS CORR.}

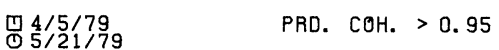

PRD. $\mathrm{COH}>0.95$

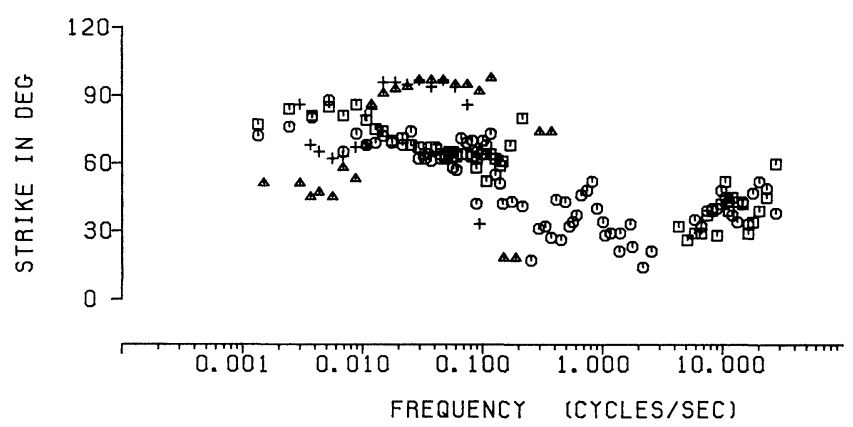

Fig. 7. Directions of axes of maximum apparent resistivity (degrees from north) determined for individual frequency bands during four separate soundings.

mencing about 1973 this uplift spread rapidly eastwards to cover an area of more than $80,000 \mathrm{~km}^{2}$. Between 1974 and 1976, the central and northern parts of the uplift began to subside. Rungle and Thatcher (1979) have proposed that relaxation of residual stresses associated with this subsidence occurred by means of crustal pore fluid diffusion. The increase in minimum apparent resistivity which we observed would be consistent with crustal pore fluids migrating out of the region thereby raising the overall crustal resistivity.

It is interesting to note in Fig. 5 the apparent return of the strike direction to about $90^{\circ}$, commencing in August, 1979. This is accompanied by a decrease in minimum apparent resistivity back to about $4 \mathrm{ohm}-\mathrm{m}$. The data for November, 1979 are rather noisy but tend to confirm this trend. It will be of interest to compare this result with up to date leveling data when this becomes available to see whether it can be correlated with a further stage of uplift.

Shown in Fig. 8 are the results of analysis of the $8 \mathrm{~Hz}$ data for the period 1978 to 1979 . The most consistent long term trend is a steady increase in the maximum resistivity phases, from about $30^{\circ}$ in mid 1978 , to about $60^{\circ}$ at the end of 1979 . It is interesting to note that there is no observable decrease in either the maximum or minimum resistivities associated with the heavy rains which occurred at the beginning of 1979. There is also a steady downward trend in $\theta_{\max }$, apart from the value in August of 1979 , which has a rather low predictability and may be biased by noise. The decreases in maximum and minimum apparent resistivity that occur in the latter part of 


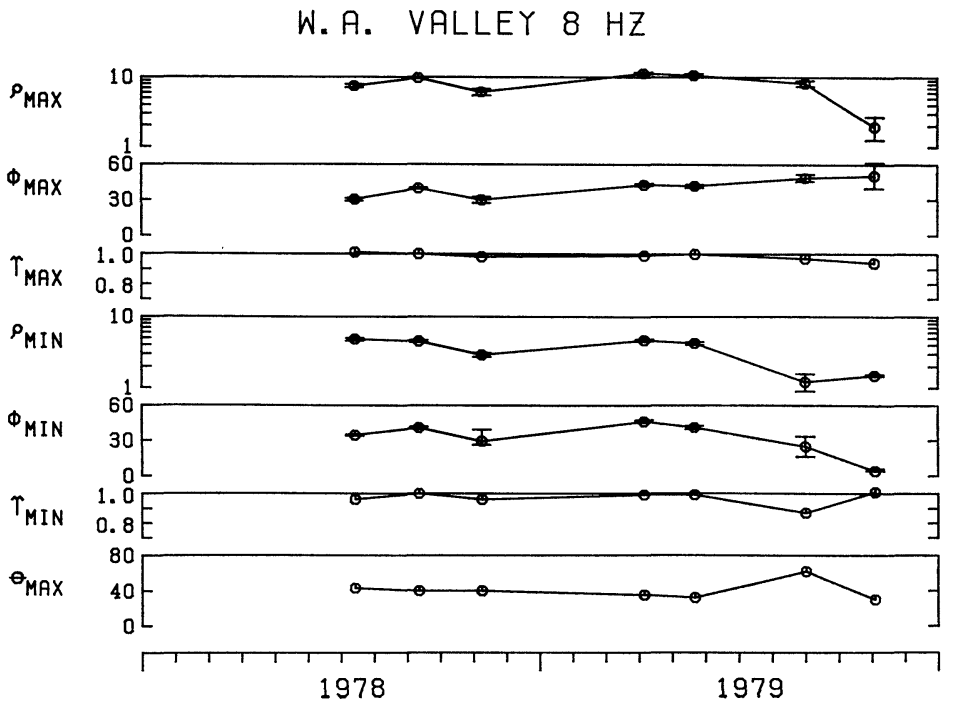

Fig. 8. Same quantities as those shown in Fig. 5 calculated at a frequency of $8 \mathrm{~Hz}$ for West Antelope Valley, 1978-1979.

1979 may be real, but data having higher predictabilities is really required to confirm this trend.

\section{LYTLE CREEK 26 SEC}

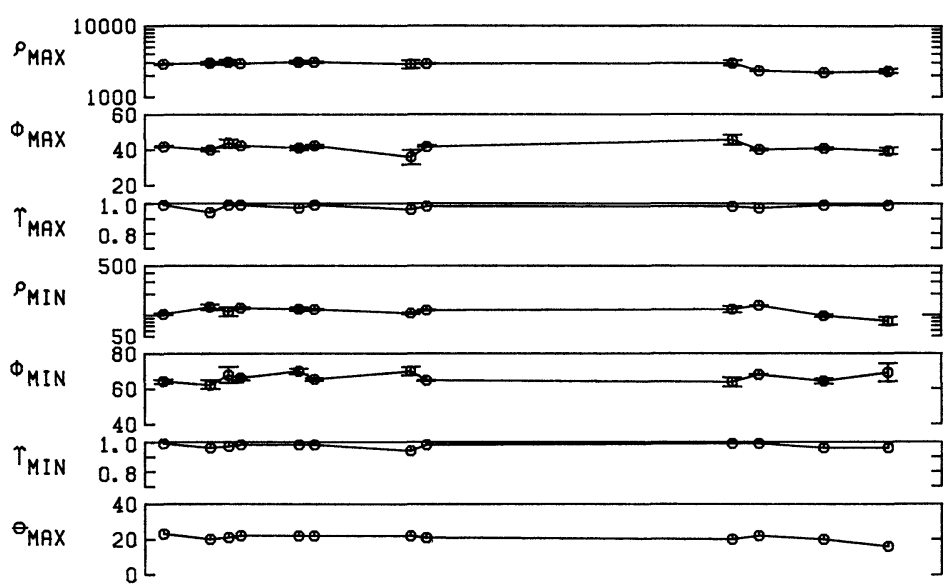

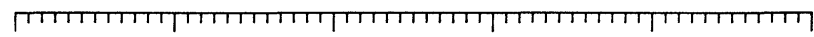

$\begin{array}{lllll}1975 & 1976 & 1977 & 1978 & 1979\end{array}$

Fig. 9. Apparent resistivity data at a period of $26 \mathrm{sec}$ for the Lytle Creek site. Notation is the same as in Fig. 5. 


\subsection{Lytle Creek}

This site lies at the northern end of the San Jacinto Fault (Fig. 1), approximately $10 \mathrm{~km}$ west of the San Andreas Fault. Results at a period of $26 \mathrm{sec}$ are shown in Fig. 9. The maximum apparent resistivities remain fairly constant up to August of 1978 where they decrease by about $20 \%$. The minimum apparent resistivities show a similar type of variation in addition to a slight rise during the middle of 1975 . The phases are rather scattered, especially those of the minimum resistivities. The scatter is in most cases within the calculated limits of error in these quantities. The direction of the maximum resistivity axis, $\theta_{\max }$, varies no more than about $5^{\circ}$ throughout the entire period.

Shown in Fig. 10 are the complete sounding curves measured in August, 1975 and in April, 1979. Although the 1979 data show some scatter, especially at the lowest frequencies, the 1979 maximum resistivity values are all generally lower than the 1979 estimates by about $20 \%$. It is possible that some of the observed differences could be due to relative calibration errors between the two systems as a $5 \%$ calibration error in a given component could result in as much as a $10 \%$ error in the resulting apparent resistivities. At frequencies above $0.5 \mathrm{~Hz}$, the two sets of data diverge rather dramatically with both the minimum and maximum apparent resistivities measured in 1975 increasing, while the 1979 values decrease. We have considered several possible explanations for this divergence between the two data sets. The first is that the frequency responses assumed for one of the two magnetotelluric systems used were in

\section{LYTLE CREEK BIAS CORR \\ O 4/4/79 *8/5/75 PRD. COH. $>0.95$}

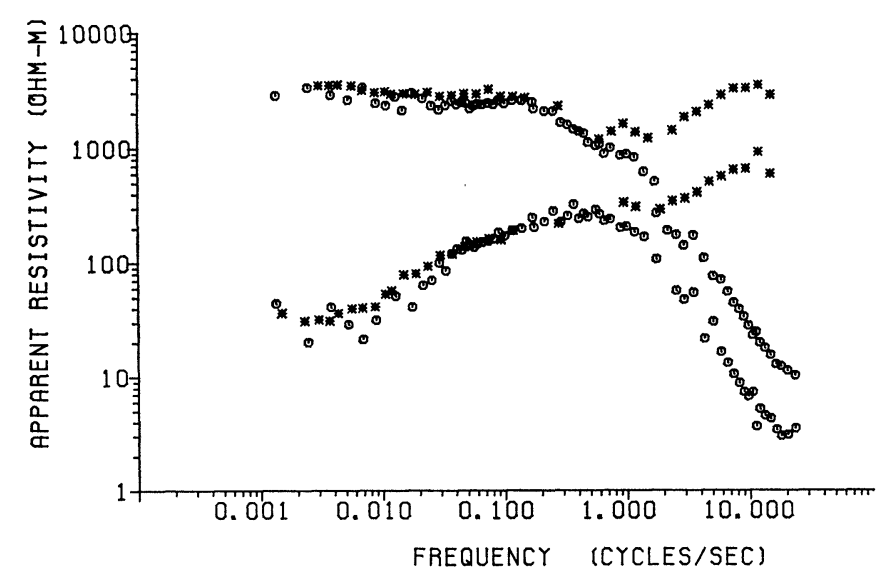

Fig. 10. Apparent resistivities observed at Lytle Creek in both August, 1975 and April, 1979. 
error. The response of our cryogenic magnetometer is flat up to about $50 \mathrm{~Hz}$ where it begins to decrease due to the presence of high frequency eddy current shields around each sensor. The frequency responses of the amplifiers used are in principle the same for both electric and magnetic fields. We measured the frequency response of both our magnetometer as well as the amplifying electronics and found them to be very close to the expected values. We therefore have no reason to suspect an error of this magnitude in the present system. Unfortunately, the earlier coil system used by REDDY et al. (1976) was returned to Canada in 1977. Until we can compare the two systems together we must therefore be somewhat cautious in interpreting changes in the high frequency data which have occurred between the two data sets. However, it is worthwhile to consider actual changes in earth resistivity which could account for the observed differences.

We assumed that the maximum apparent resistivities at this site could be modeled using a one-dimensional two-layer model (PATRICK and Bostick, 1969), the lower layer having a resistivity of $3,000 \mathrm{ohm}-\mathrm{m}$. The data could then be fitted reasonably well with an upper layer having a resistivity of $10 \mathrm{ohm}-\mathrm{m}$ and a thickness of $10 \mathrm{~m}$. These seem reasonable values for weathered soil overlying the crystalline metamorphic rocks which underlie this site. However, to explain the 1975-1976 data the upper layer would have to have had a resistivity at least two orders of magnitude higher. It is not unreasonable to propose a higher resistivity for such a layer prior to 1978, as a prolonged drought was ended by extremely heavy rainfall in this area early in 1978,

\section{LYTLE CREEK $8 \mathrm{HZ}$}

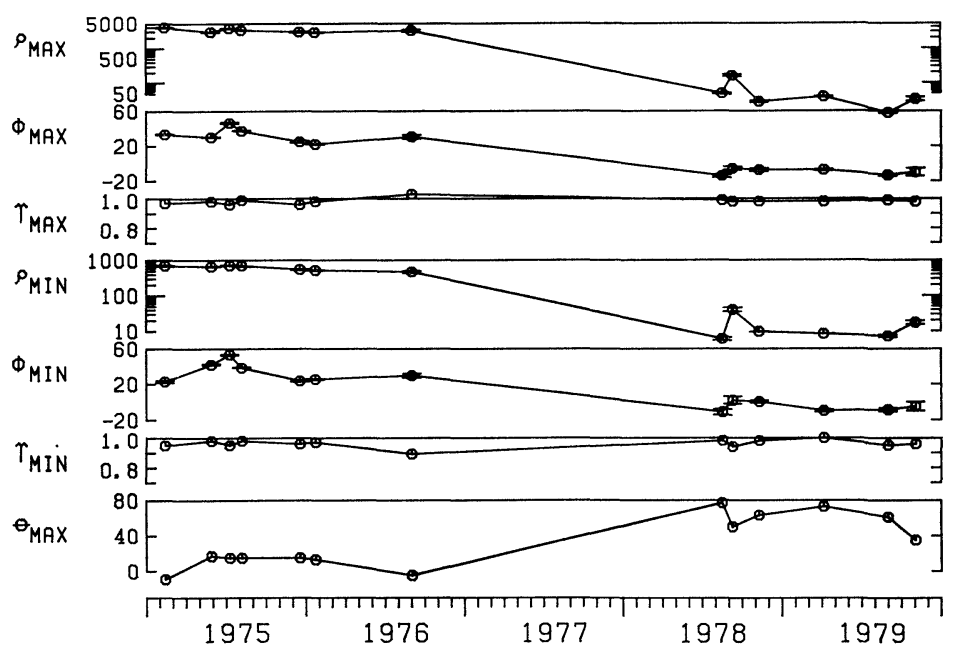

Fig. 11. Apparent resistivity variations at $8 \mathrm{~Hz}$ for the Lytle Creek site. Notation is the same as in Fig. 5. 
and also in 1979. It may also be possible to explain the data with smaller changes in complex three-dimensional resistivity structures, such as numerous fractures that have become water saturated. Such fractures are undoubtedly present in complex tectonic areas such as this.

Shown in Fig. 11 are the $8 \mathrm{~Hz}$ results for Lytle Creek. The most dramatic feature is the drop discussed above which occurred between 1976 and 1978. The short term variations are probably associated with local fluctuations in the water table. The extremely high resistivities of the crystalline basement at this site would tend to make the apparent resistivities extremely sensitive to such fluctuations.

\subsection{China Flats}

This site is located to the west of Los Angeles, approximately $50 \mathrm{~km}$ south of the San Andreas Fault (Fig. 1). Because of its close proximity to densely populated areas, it has proved to be the noisiest of the three sites. The $26 \mathrm{sec}$ apparent resistivity data appear in Fig. 12. The maximum and minimum apparent resistivities both remain fairly stable apart from a few isolated points having large errors and relatively low predictabilities. There is some suggestion of a drop in the values of both these quantities between August and November of 1978, i.e., after the Santa Barbara Channel earthquake of August 13, 1978. However, the rather large errors on the points make this drop of only marginal significance. The largest variations occur in the minimum resistivity phase, but again the rather large errors suggest that the data may be biased by noise.

CHINA FLATS 26 SEC

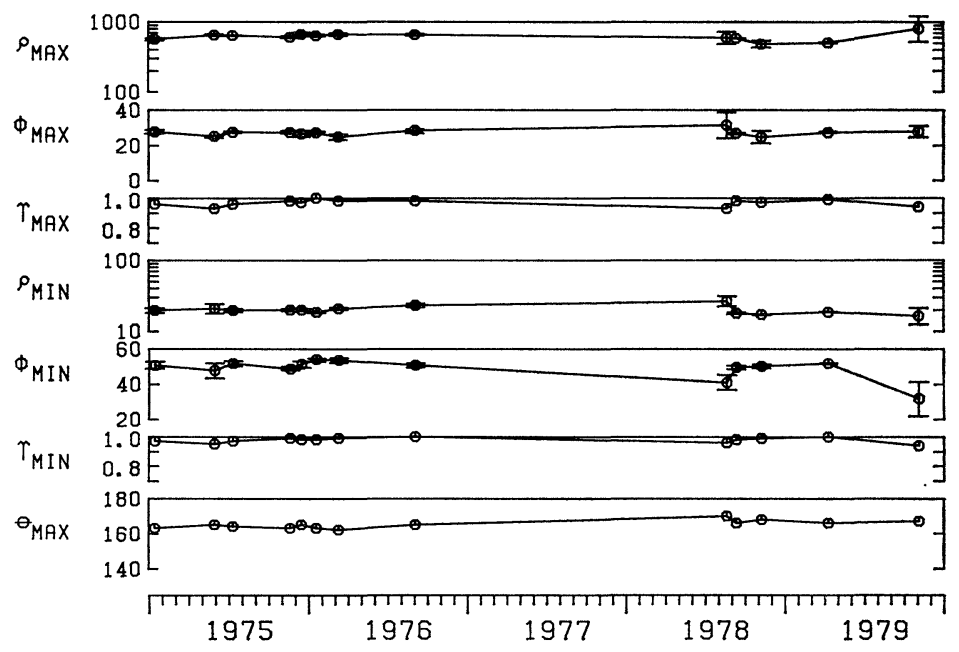

Fig. 12. China Flats apparent resistivity variations at a period of $26 \mathrm{sec}$. 


\section{CHINA FLATS BIAS CORR}

O9/11/78 * 7/10/75 PRD. COH. $>0.95$

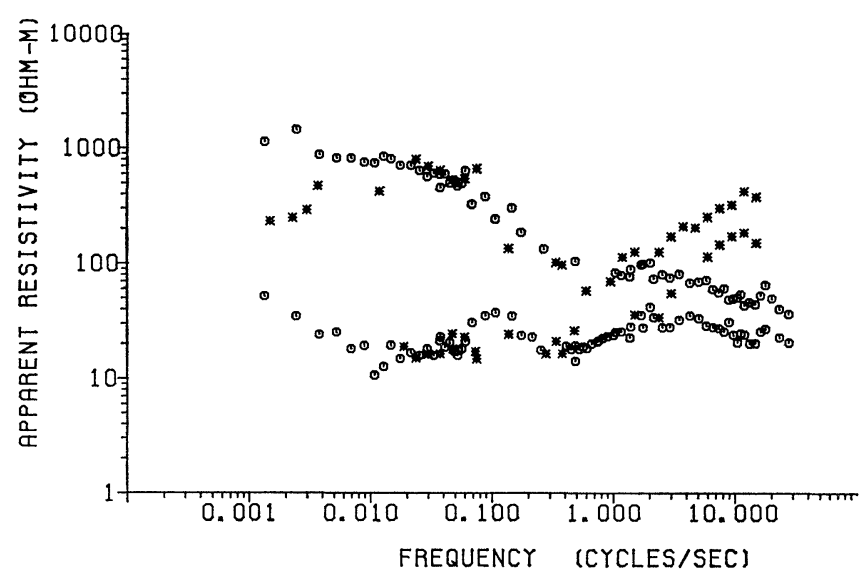

Fig. 13. Apparent resistivities observed at China Flats in July, 1975 and September, 1978.

Shown in Fig. 13 are the complete sounding curves obtained in July, 1975 and September, 1978. Agreement between the two data sets is reasonable between 0.01 and $1 \mathrm{~Hz}$ although much of the data is scattered. The lower values of the 1975 maximum resistivities which occur below $0.01 \mathrm{~Hz}$ are not considered significant as the corresponding minimum resistivities had low predictabilities, making the strike directions and consequently the maximum values of apparent resistivity unreliable. Other low frequency data collected in 1975-1976 having higher predictabilities agreed fairly well with the 1978 values shown in Fig. 11. The high frequency data in Fig. 13 shows a similar divergence to the Lytle Creek data, although less pronounced. The difference between the maximum apparent resistivities determined at $10 \mathrm{~Hz}$ in 1975 1976 versus those determined in 1978-1979, is about a factor of 8 at this site. Such changes could be reasonably accounted for by the heavy rains which occurred in early 1978 reducing the resistivity of a thin soil layer.

Shown in Fig. 14 are the $8 \mathrm{~Hz}$ results for the China Flats site. Again, the decrease occurring between 1976 and 1978 is the most pronounced feature. However, at this site, the maximum resistivities tend to increase from the minimum value in July of 1978 value, which is the type of behavior to be expected as the ground dried out following the heavy rainfall in the early part of 1978. Note that there is little difference between the results obtained in July and those in August of 1978. The Santa Barbara event $\left(M_{L}=5.2\right)$ occurred $130 \mathrm{~km}$ to the west of the China Flats site during this period. Although the August data was rather noisy and no $26 \mathrm{sec}$ data was obtained in July, we observed no significance between the two sets of results at any of the 


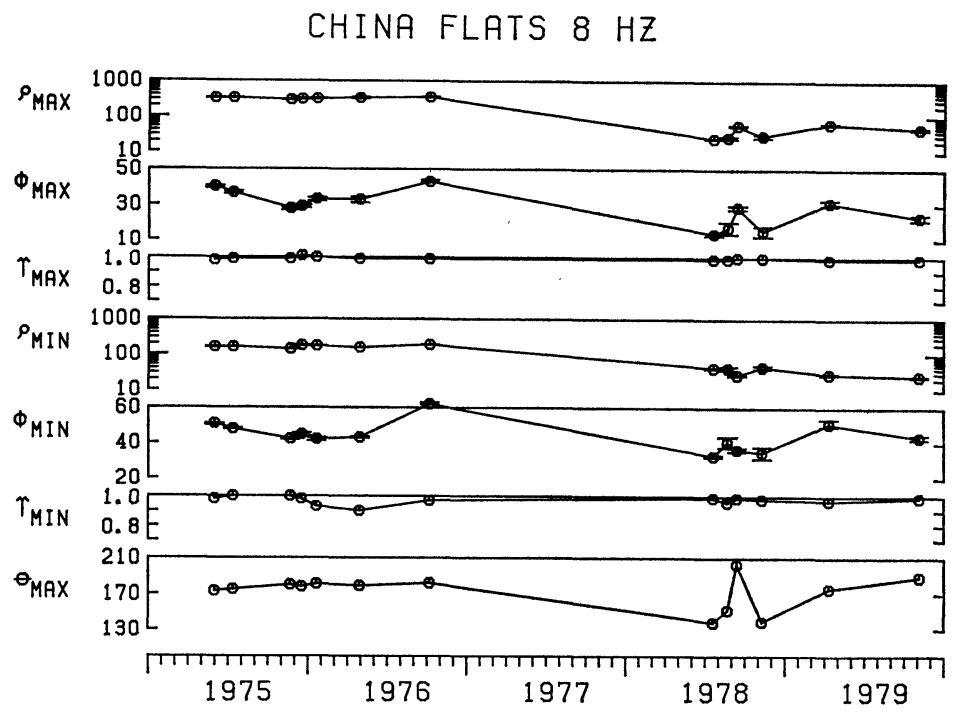

Fig. 14. China Flats apparent resistivity variations at a frequency of $8 \mathrm{~Hz}$.

frequencies for which reliable estimates were obtained. The results for September, 1978 are clearly anomalous, with the maximum resistivity increasing by a factor of 2 and $\theta_{\max }$ by more than $60^{\circ}$. However, as we observed earlier, individual results such as this should be viewed with some suspicion, especially since and anomalous result also appears in the $8 \mathrm{~Hz}$ Lytle Creek data for September, 1978 (Fig. 11). It is possible that the source field was extremely non-uniform during the two days the measurements were made at both of these sites causing bias in both of the estimates.

\section{Discussion}

So far, we have not attempted to correlate any of the observed changes in the magnetotelluric data with seismicity in the area. The seismicity is summarized in Fig. 15 in which epicenters of earthquakes having magnitudes $\geq 3.5$, and occurring between 1975 and 1979 are plotted. The site at which the largest variations occurred, West Antelope Valley, lies in a region that has remained relatively aseismic during the period of this study. The changes cannot therefore be correlated with seismicity, at least in the immediate vicinity of the site. Events do occur within $50 \mathrm{~km}$ of the other two sites. However, detailed examination of the times and locations of these events has failed to show any clear correlations, mainly because the variations in resistivities at these two latter sites are rather subtle and poorly defined through lack of data at critical times.

Our conclusions regarding repeatability in these types of measurements is that 


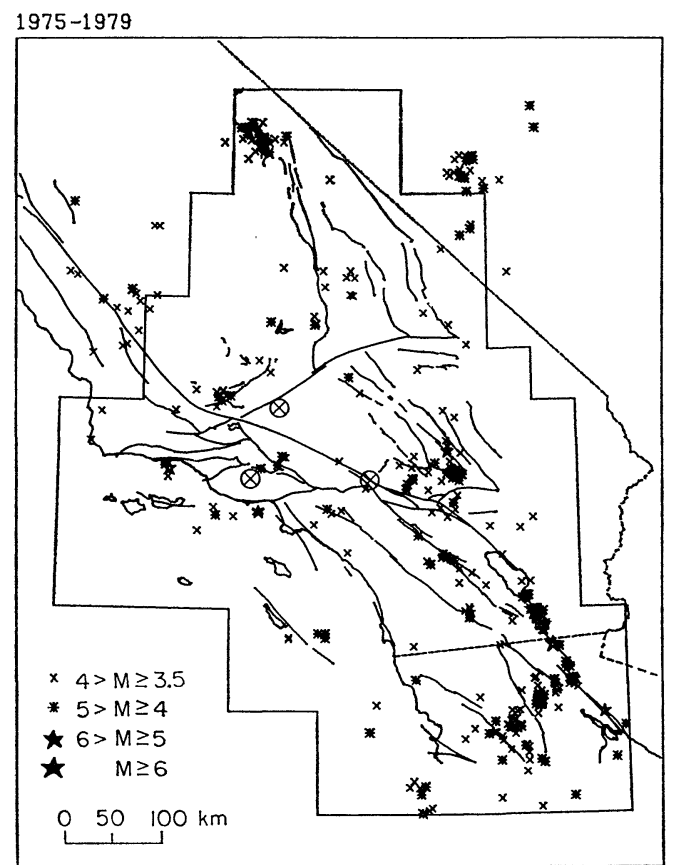

Fig. 15. Locations of earthquakes $(M \geq 3.5)$ which occurred in Southern California, 19751979 , relative to the major fault systems and the magnetotelluric sites.

variations of greater than $20 \%$ could easily be resolved using the measurement and analysis techniques described above. However, variations in high frequency apparent resistivity data may be associated with local water table fluctuations, especially in areas such as Lytle Creek, where the basement resistivities are very high.

This research was supported by California Institute of Technology President's Fund. Contribution No. 3359, Division of Geological and Planetary Sciences, California Institute of Technology, Pasadena, California 91125.

\section{REFERENCES}

Allsopp, D. F., M. D. Burke, D. Rankin, and I. K. RedDy, A wide band digital magnetotelluric recording system, Geophys. Prospect., 22, 272-278, 1974.

BARSUKOV, O. M., Variations of electric resistivity of mountain rocks connected with tectonic causes, Tectonophysics, 14, 273-277, 1972.

Bendat, J. S. and A. G. Piersol, Random Data: Analysis and Measurement Procedures, John Wiley and Sons, Inc., New York, 1971.

BRACE, F. W. and A. S. ORANGE, Electrical resistivity changes in saturated rocks during fracture and frictional sliding, J. Geophys. Res., 73, 1433-1445, 1968. 
Castle, R. O., J. P. Church, and M. R. Elliot, Aseismic uplift in Southern California, Science, 192, 251-253, 1976.

Castle, R. O., M. R. Elliot, and S. H. Wood, The Southern California uplift (abstract), EOS Trans., $A G U, \mathbf{8 5}, 495,1977$.

Jupp, D. L. P., Estimation of the magnetotelluric impedance functions, Phys. Earth Planet. Inter., 17, 75-82, 1978.

Mazzella, A. and H. F. Morrison, Electrical resistivity variations associated with earthquakes on the San Andreas Fault, Science, 185, 855-857, 1974.

Otnes, R. K. and L. Enochson, Digital Analysis of Time Series, John Wiley and Sons, Inc., New York, 1972.

Patrick, F. W. and F. X. Bostick, Jr., Magnetotelluric modeling techniques, Tech. Rep. No. 59, 100 pp., Electrical Geophysics Laboratory, University of Texas at Austin, 1969.

PricE, A. T., The theory of magnetotelluric methods when the source field is considered, J. Geophys. Res., 67, 1907-1918, 1962.

REDDY, I. K. and D. RANKIN, Coherence functions for magnetotelluric analysis, Geophysics, 39, 312-320, 1974.

Reddy, I. K., R. J. Phillips, J. H. Whitcomb, D. M. Cole, and R. A. Taylor, Monitoring of timedependent electrical resistivity by magnetotellurics, J. Geomag. Geoelectr., 28, 165-178, 1976.

Rundle, J. B. and W. Thatcher, A stress relaxation model for the Southern California uplift (abstract), EOS Trans., AGU, 60, 321, 1979.

Sims, W. E., F. X. Bostick, Jr., and H. W. SмIтH, The estimation of magnetotelluric impedance tensor elements from measured data, Geophysics, 36, 938-942, 1971.

SwifT, C. M., A magnetotelluric investigation of an electrical conductivity anomaly in the Southwestern U. S., Ph. D. Thesis, Mass. Inst. of Technology, 211 pp., 1967.

VozofF, K., The magnetotelluric method in the exploration of sedimentary basins, Geophysics, 37, 98-141, 1972.

White, R. E., The estimation of signal spectra and related quantities by means of the multiple coherence function, Geophys. Prosp., 21, 660-691, 1973. 